\title{
AJUSTE DE LA PRESIÓN ANULAR EN POZOS CON BLOQUEO POR GAS PARA OPTIMIZACIÓN DE PRODUCCIÓN EN EL CAMPO CIRA INFANTAS
}

Rogelio Márquez Quintero', Camilo Martínez Lamus², Samuel Arévalo Garzón ${ }^{3 *}$ Erik Montes Páez, Félix cepeda Gómez ${ }^{5}$

* A quien debe dirigirse la correspondencia.

\section{RESUMEN}

La industria petrolera se ha caracterizado a lo largo de la historia por la evolución en la forma de desarrollo de los campos. Antiguamente, cuando los campos dejaban de ser económicamente viables debido a las bajas tasas de producción o a los altos costos de extracción, se abandonaban sin consideración. Hoy en día, existen ciertas alternativas para mejorar la producción a un costo viable. En el siguiente artículo se expone la metodología propuesta para contrarrestar un problema denominado "bloqueo por gas" en el campo La Cira-Infantas del Valle Medio del Magdalena, el cual surge cuando la presión de fondo fluyente es menor a la presión de burbuja, lo cual genera flujo multifásico en la cara de la formación, causando el descenso de las tasas de producción. Se elaboró una metodología práctica analizando y teniendo en cuenta el comportamiento histórico de producción y se comparó con un modelo de simulación en el software Pipesim. Finalmente se analizaron los resultados obtenidos y se propusieron los criterios para establecer los pozos candidatos a ser sometidos al mismo procedimiento.

Palabras clave: anular, presión, bloqueo por gas, producción, optimización

\section{ADJUSTING OF THE ANNULAR PRESSURE IN WELLS BLOCKED BY PRESENCE OF GAS OPTIMIZE THE PRODUCTION IN CIRA INFANTAS FIELD}

\begin{abstract}
The oil and gas industry has been traditionally recognized throughout history due to its facility to evolve in topics related to resources exploitation and development. Previously, an oil field could be considered non-profitable when it reaches low oil production rates or high operational expenses resulting in its abandonment. A useful starting point for this article is the study of the reservoir fluids behavior. According as a definite volume of fluids is extracted from the reservoir, the system progressively lost energy until the static bottom-hole pressure equals the saturation pressure. At this point, it is released the first bubble of gas and the multiphasic flow appears carrying out particular issues such as gas blockage that directly affects the oil productivity index. In this article, it is exposed a

1. Ingeniero de Petróleos, Universidad Industrial de Santander, Occidental de Colombia. Email: roger.1009@ hotmail.com

2. Ingeniero de Petróleos, Universidad Industrial de Santander. Email: kamilo_0993@hotmail.com

3. Ingeniero de Petróleos, Grupo de Investigación de Optimización de la Producción, Universidad Industrial de Santander. Email: samuel.arevalo.g@hotmail.com

4. Magister en Ingeniería de Hidrocarburos, Grupo de Investigación de Modelamiento de Pozos, Escuela de Ingeniería de Petróleos, Universidad Industrial de Santander. Email: erimonpa@uis.edu.co

5. Especialista en Producción de Hidrocarburos, Occidental de Colombia, Departamento de Ingeniería, Bogotá, Colombia. Email: felix_cepeda@oxy.com
\end{abstract}


detailed methodology to diminish that concrete problem exhibited in several oil wells from the Cira-Infantas which significantly strikes the well performance. In fact, it was made up a practical methodology taking into count the fluids properties variations and the historic production performance in order to compare the results obtained against a thorough analysis generated by computational modeling in Pipesim flow simulator. Finally, the acquired results were meticulously processed and there were proposed several criteria to identify the suitable wells to be submitted for the same procedure.

Keywords: annular, pressure, gas blockage, production, optimization

\section{INTRODUCCION}

La Cira-Infantas es un campo maduro ubicado en la cuenca del valle medio del Magdalena, tiene una producción promedio de 40,000 barriles de aceite por día y cuenta con inyección de agua como mecanismo de recobro secundario. Dentro de las estrategias para aumentar la producción se ha implementado el cañoneo de zonas productoras más profundas como son las unidades de $\mathrm{C}$ inferior y $\mathrm{D}$, estas zonas se caracterizan por tener altas presiones originales y cortes de agua bajos ya que aún no cuentan con soporte de inyección de agua.

El pozo CIRA-M, ubicado en el área de Cira Este y abierto en las unidades C4-Cb y D1, presentó una caída de producción en noviembre de 2014 de aproximadamente 300 barriles por día, y desde entonces ha tenido una producción promedio de 80 barriles por día. Esta caída de producción coincide con la conexión del pozo a la red de recolección de gas de anulares, es decir, con una disminución en el valor de la presión de anular y por consiguiente en la presión de fondo fluyente, lo que pudo ocasionar una alta liberación de gas en la cara de la formación debido a que el fluido del pozo tiene una alta presión de burbuja. Este problema se denomina bloqueo por gas y tiene un efecto negativo en la productividad de la formación pues afecta directamente la permeabilidad relativa del aceite.

\subsection{BLOQUEO POR GAS EN LA CARA DE LA FORMACIÓN}

Durante la producción de yacimientos de aceite, la caída de presión causa la formación de una segunda fase, lo que conlleva a un régimen de flujo bifásico. Esto, junto con otros factores como la naturaleza del fluido, las propiedades de la roca y del yacimiento, y los gradientes de presión impuestos, pueden afectar negativamente la productividad del yacimiento.

En los yacimientos de aceite, cuando la presión del fondo del pozo (BHP) cae por debajo de la presión del punto de burbuja, la formación de una segunda fase se produce en la zona cercana al pozo, lo que resulta en flujo multifasico. En zonas alejadas a la cara del pozo donde la presión está por encima del punto de burbuja sólo la fase líquida está presente (aceite sub-saturado).

Se pueden identificar tres regiones principales en el yacimiento a medida que nos alejamos del pozo, como se ilustra en la Figura 1. La Región 1 se genera cuando las caídas de presión de fondo de pozo están por debajo de la presión del punto de burbuja y el volumen de gas liberado es lo suficientemente alto para superar la saturación de gas residual de modo que el gas puede fluir. Por lo tanto, en esta región cerca del pozo, se produce flujo de múltiples fases (gas y líquido).

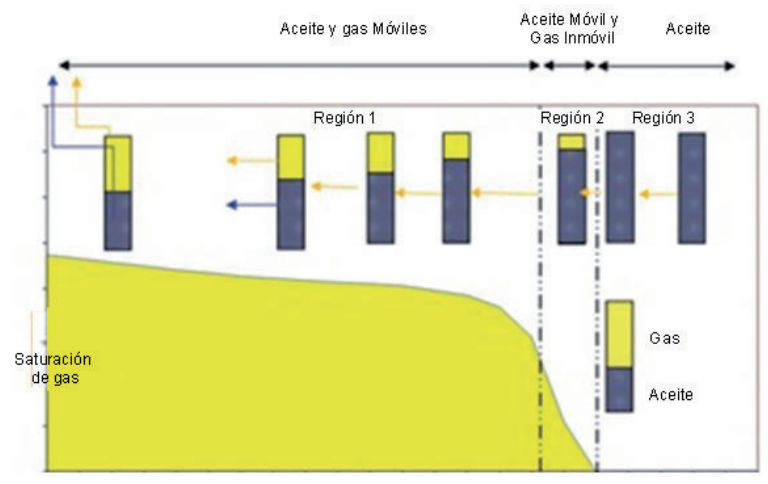

Figura 1. Saturación de gas por regiones en un pozo de aceite volátil con BHP por debajo de la presión de burbuja

Fuente: Los autores

La Región 2 es donde la presión cae por debajo de la primera presión del punto de burbuja, es decir, donde las burbujas de gas se empiezan a formar y el volumen de gas liberado en esta región está por debajo de la saturación de gas crítica, lo que resulta en una fase de gas inmóvil. En la región 2 sólo fluye la fase líquida. Finalmente, se encuentra la Región 3 , donde la presión en el yacimiento excede el punto de burbuja y existe aceite sólo como fase líquida móvil. La identificación de esta región es similar a la de los yacimientos de gas condensado con la única diferencia de que las fases se invierten (Torres, et al., 2010), (Torres, 2011). 


\subsection{CAUSAS}

La sensibilidad de un yacimiento a cualquiera de los fenómenos descritos anteriormente y cómo influye en la tasa de producción, depende de varios factores. Algunos de estos son: comportamiento de fase, régimen de flujo, fuerzas interfaciales entre fluidos, número capilar, heterogeneidad del yacimiento, propiedades de los fluidos y la roca, mojabilidad y fuerzas gravitacionales.

Por otra parte, el manejo de las condiciones operacionales del pozo influye de manera sustancial, ya que determina en qué parte del diagrama de fases se encuentra el fluido y por tanto, las fases que están presentes a dichas condiciones en el yacimiento. Tener un control de la presión de fondo es primordial para evitar este problema, y dicha variable depende de la presión de anular y de la presión hidrostática ejercida por la columna de líquido almacenada en el espacio anular denominada sumergencia efectiva. (Figura 2).

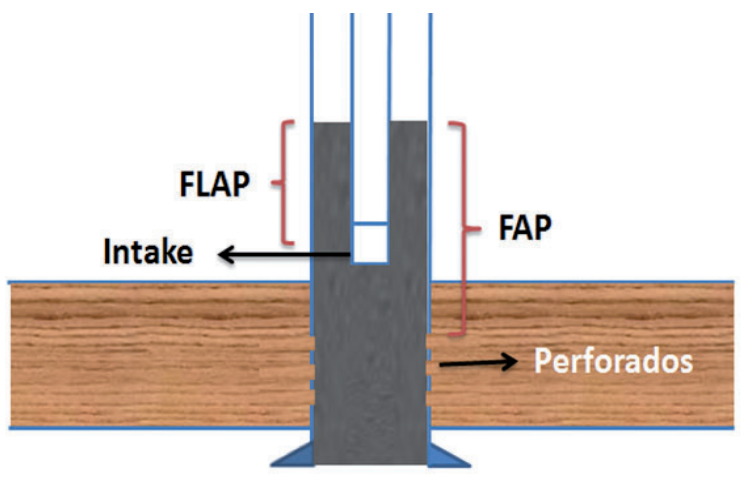

Figura 2. Sumergencia efectiva Fuente: Los autores

La sumergencia puede ser medida sobre el tope de perforados (FAP) o sobre la entrada de la bomba de subsuelo (FLAP), estos datos son tomados mediante registros acústicos Sonolog.

\subsubsection{ALTERNATIVAS PARA MITIGAR EL BLOQUEO POR GAS}

Para restaurar las tasas de producción de aceite después de una declinación debido al bloqueo por gas, se han propuesto varios métodos, los cuales se describen brevemente a continuación:

\subsubsection{FRACTURAMIENTO HIDRÁULICO}

Sanni y Gringarten (2008) modelaron un pozo de aceite volátil produciendo a una presión de fondo fluyente por debajo de la presión de burbuja. A través de un modelo de simulación unidimensional de un pozo productor en un yacimiento composicional comprobó que con fracturas hidráulicas y pozos horizontales, se puede aumentar la eficiencia en la productividad debido a la mitigación del bloqueo por gas.

\subsubsection{AGENTES QUÍMICOS}

En yacimientos donde una capa de aceite delgada se encuentra debajo de una capa de gas, la producción puede ser mejorada mediante la generación de una barrera de espuma en la formación. Esta barrera puede aumentar sustancialmente las tasas de aceite reduciendo o retardando la conificación del gas. La espuma confinada dentro de la red de espacios porosos de la roca consiste de una película delgada de líquido que se extiende a través de los poros, lo cual hace que la fase gaseosa sea discontinua (Hanssen \& Haugum, 1991).

\subsubsection{SOLUBILIZACIÓN DEL GAS POR INCREMENTO DE PRESIÓN EN FONDO}

Otra alternativa para contrarrestar el efecto del bloqueo por gas es cambiar las condiciones de presión del fluido en fondo con el fin de solubilizar el gas que se encuentra en la cara de la formación, para esto es necesario que la presión alcanzada sea mayor a la presión de burbuja.

\section{METODOLOGÍA DESARROLLADA}

Para desarrollar una metodología que permitiera contrarrestar los efectos del bloqueo por gas en la cara de la formación fue necesario establecer un modelo teórico y un modelo práctico aplicados a un pozo piloto para luego comparar los resultados obtenidos. 


\subsection{COMPORTAMIENTO HISTÓRICO Y ANÁLISIS DE VARIABLES DE PRODUCCIÓN DEL POZO PILOTO}

El pozo piloto CIRA-M se encuentra ubicado en el área Cira Este, abierto en las unidades de $\mathrm{C} 4$ y D1 con una profundidad total de 4.578 pies. El pozo se perforó en diciembre de 2013 y fue completado con un sistema de levantamiento electro-sumergible. (Figura 3).

Mediante análisis de laboratorio PVT se identificó que el fluido producido es un aceite negro, el cual registró una presión de yacimiento de 1.670 psi y una presión de burbuja de 1.516 psi. Este último valor es considerablemente alto en contraste al promedio del campo y es debido a que las unidades $\mathrm{D}$ son arenas nuevas que no se habían explotado.

Utilizando los datos PVT y mediante el uso de la herramienta software Hysys se obtuvo el diagrama de fases del fluido del pozo, en el que se puede observar que las condiciones iniciales de presión y temperatura del yacimiento están muy cerca de la envolvente, es decir, con una pequeña caída de presión habrá liberación de gas en el reservorio. (Figura 4).

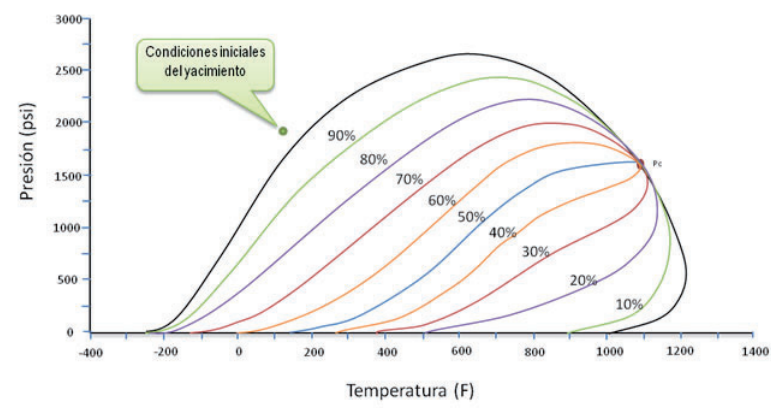

Figura 3. Diagrama de fases del fluido del pozo CIRA-M

Fuente: Los autores

El pozo CIRA-M fue puesto en marcha en febrero de 2014 y en marzo de ese mismo año fue sometido a la primera prueba de producción, obteniendo como resultado 200 barriles de fluido por día con un corte de agua del $1 \%$. Este comportamiento se mantuvo durante los siguientes 6 meses en donde el sistema de bombeo electro-sumergible fue configurado a una frecuencia promedio de $52 \mathrm{~Hz}$.

Posteriormente, el sistema de levantamiento fue ajustado a la máxima frecuencia de operación permitiendo alcanzar un pico máximo de producción de 430 barriles por día en el mes de septiembre del 2014 y 400 barriles por día en octubre, conservando un corte de agua no superior al 2\%. Sin embargo, en el mes de noviembre el pozo registró una disminución considerable en la producción hasta alcanzar un valor promedio de 80 barriles por día. (Figura 5).

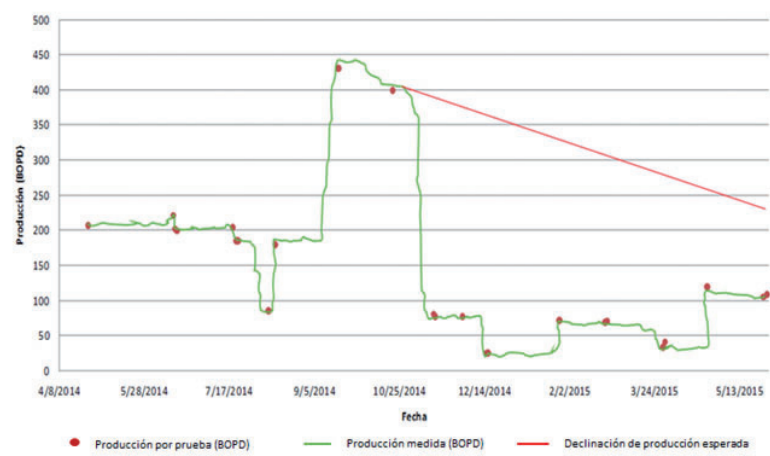

Figura 4. Histórico de producción del pozo CIRA-M

Por otra parte, se puede observar que inicialmente el pozo operaba con una presión de anular (CHP) de aproximadamente 200 psia cuando el comportamiento de la producción era estable y los valores de presión a la entrada de la bomba (PIP) eran altos aunque variables, ya que se evidencian variaciones de frecuencia que afectaban la sumergencia del pozo (Figura 6). Sin embargo, se registró una caída considerable en la presión de anular dando como resultado una disminución en la producción, a pesar que se mantenía un rango de valores elevados en la PIP y por consiguiente de presión de fondo fluyente.

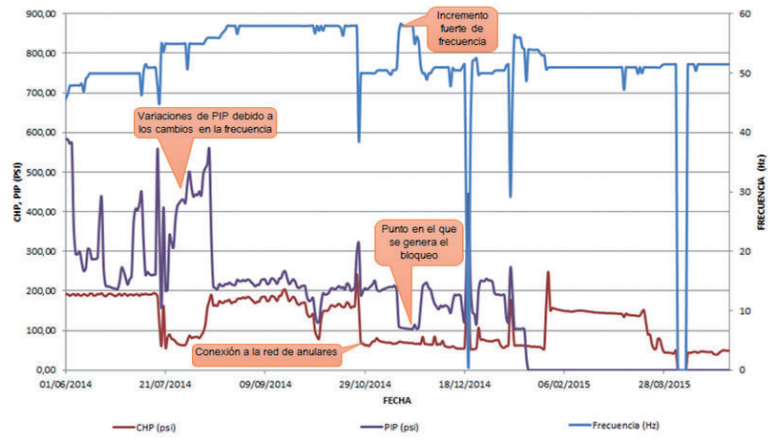

Figura 5. Comportamiento de las variables CHP, PIP y frecuencia de la bomba del pozo CIRA-M Fuente: Los autores

Posteriormente, se configuró la frecuencia del sistema electro-sumergible a $58 \mathrm{~Hz}$, alcanzándose el máximo potencial de producción del pozo y una disminución considerable en la sumergencia como se observa en los valores de PIP, razón por la cual se concluyó que la presión de fondo fluyente (BHP) dependía prácticamente de la presión de anular (CHP). 
Durante el mes de noviembre se presentó un descenso notable de la presión de casing, ya que el pozo fue conectado a la línea de recolección de gas de anulares. Sin embargo, como se obtuvo una disminución en la frecuencia de operación de la bomba el valor de PIP se mantuvo estable. Luego a mediados de noviembre nuevamente se lleva la frecuencia a $58 \mathrm{~Hz}$ lo que implicó que el pozo se quedara sin sumergencia, es decir, la presión de fondo fluyente alcanzó el valor más bajo registrado, lo que causó una gran liberación de gas en la cara de la formación y dio lugar al problema denominado "bloqueo por gas". Fue en este punto en donde la producción del pozo cayó drásticamente y desde entonces a pesar de que se disminuyó la frecuencia de la bomba no se pudo recuperar.

\subsection{PROCEDIMIENTO PROPUESTO PARA CONTRARRESTAR EL BLOQUEO POR GAS}

Luego de analizar las variables de producción se decidió que la mejor forma de evitar el bloqueo por gas es controlando la presión de anular (CHP) mediante la instalación de una válvula choque para restringir el flujo a través de la línea de recolección de gas de anulares, mediante esta aplicación básica es posible encontrar el valor óptimo que permita tener la menor liberación de gas y la mayor producción de aceite. Por lo tanto, antes de ajustar el rango de presiones en la línea de anular, es necesario "desbloquear el pozo", es decir, tratar de solubilizar el gas que se encuentra en la cara de la formación para mejorar la permeabilidad relativa del aceite y tener un mayor índice de productividad.

A continuación se describe el procedimiento propuesto luego de instalar la válvula choque:

- Apagar el equipo de fondo desde el variador de frecuencia.

- Reducir la presión de casing hasta el valor mínimo posible liberando el gas que se encuentra almacenado en el espacio anular.

- Inyectar agua al anular, previamente se deben realizar los cálculos volumétricos teniendo en cuenta la presión que se quiere alcanzar en el fondo del pozo.

- Dejar apagado aproximadamente 8 horas (Lo ideal es dejar el equipo apagado el tiempo que sea posible) para que el nivel de fluido se estabilice.

- Arrancar el equipo de bombeo electro-sumergible a una frecuencia mínima de aproximadamente $40 \mathrm{~Hz}$.
- Mantener la válvula choque cerrada para aumentar la presión de anular.

- Medir el nivel de fluidos mediante un dispositivo Sonolog, para determinar la cantidad de agua presente en el anular y el volumen absorbido por la formación.

- Aumentar gradualmente la frecuencia del equipo de levantamiento $(1 \mathrm{~Hz})$, hasta que la presión en cabeza sea suficiente alta para que el fluido entre al múltiple de producción o Manifold.

- Observar el comportamiento de la presión de anular y abrir gradualmente la válvula si la presión está por encima de 200 psia, pero controlando que la presión se estabilice y no alcance valores muy bajos.

- Tomar niveles de fluido mínimo 2 veces por día (mañana y tarde) para calcular el valor de presión de fondo fluyendo (BHP).

- Realizar mediciones de caudal conjuntamente con las variaciones de frecuencia y CHP.

- Dependiendo del comportamiento del pozo y los datos históricos establecer un rango de presión de anular (CHP) y presión de fondo (BHP) óptimo, garantizando el máximo escenario de recuperación de aceite evitando el bloqueo por gas.

\subsection{IMPLEMENTACIÓN EN EL POZO PILOTO}

Para hacer posible el ajuste de la presión de anular fue necesario modificar la configuración de la red de recolección de gases de anular, ya que ésta solo contaba con un manómetro y una válvula de bola para cerrar o abrir el paso a través de la línea (Figura 7). El arreglo que se implementó cuenta con 1 válvula de bola, 1 válvula de choque y 2 manómetros análogos ubicados antes y después de dicha restricción (Figura 8).

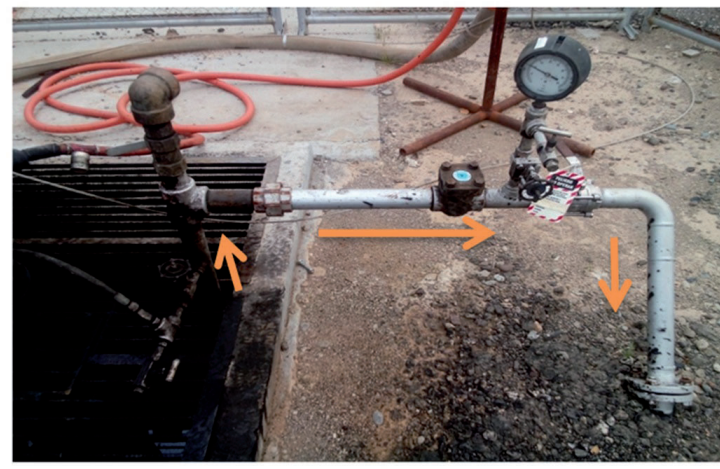

Dirección del flujo de gas de anular

Figura 6. Red de recolección de gas de anulares del pozo CIRA-M antes de ser modificada 


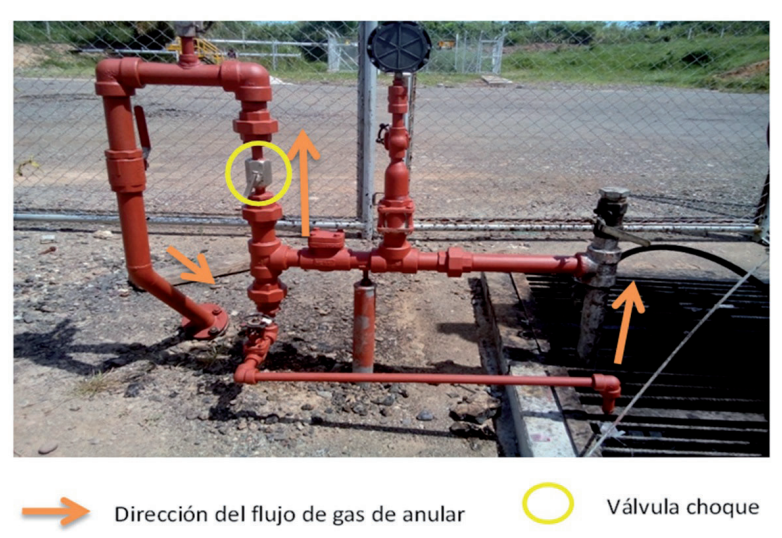

Figura 7. Red de recolección de gas de anulares del pozo CIRA-M luego de la instalación de la válvula choque

El objetivo del procedimiento propuesto es lograr solubilizar el gas que se encuentra bloqueando la cara de la formación. La hipótesis es que este problema se da debido a que el fluido del pozo tiene una presión de burbuja considerablemente alta y con pequeñas caídas de presión hay liberación de gas, lo que implica que para tratar de contrarrestar el efecto negativo del bloqueo es necesario que la presión en el fondo del pozo sea mayor a la presión de burbuja y de esta manera el gas que se encuentra en la cara de la formación pase a estado líquido.

Con la finalidad de aumentar la presión en el fondo del pozo se inyectó agua en el anular (Figura 9). Para ello, se determinó el volumen de agua necesario para alcanzar una presión mayor a la presión de burbuja en el punto medio de los perforados y el volumen necesario para llenar completamente el espacio anular.

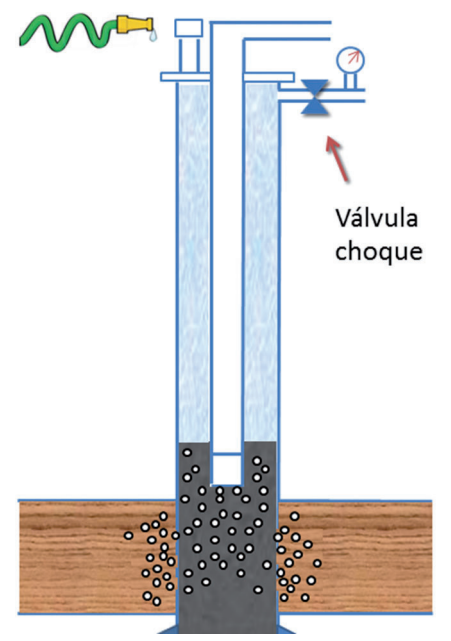

Figura 8. Inyección de agua al espacio anular
Después de finalizado el proceso de inyección de agua en el espacio anular, se apagó el equipo durante ocho horas y se tomó un nivel de fluido que dio como resultado 800 pies de sumergencia. Posteriormente, se reactivó el sistema de bombeo electro-sumergible a 40 $\mathrm{Hz}$, sin embargo, esta frecuencia no era suficiente para levantar el fluido en superficie, por lo que fue necesario aumentarla gradualmente hasta llegar a $50 \mathrm{~Hz}$, donde se alcanzó una presión en cabeza de 225 psia, suficiente para que el fluido entrara al múltiple de producción cuya presión de operación es de 220 psia. La válvula choque se mantuvo cerrada al inicio para que la presión en el anular aumentara, y luego se reguló para mantenerla en un rango entre 150 y 200 psia.

Para el cálculo de la presión de fondo fluyendo (BHP), se midieron diariamente los niveles fluidos y luego de esto se desarrollaron los cálculos correspondientes de presión hidrostática. Por otra parte, para realizar correctamente la medición del caudal de aceite producido por el pozo, fue necesario esperar que el agua inyectada retornara hasta superficie para obtener el dato real de producción.

\subsection{RESULTADOS}

Luego de aplicar el procedimiento propuesto en el pozo CIRA-M, se llevó a cabo el seguimiento a las variables de presión de tubería (THP) y presión de anular (CHP) mediante el uso de softwares de monitoreo y control.

Debido a la falta de personal en campo durante las horas de la noche, no fue posible manipular la válvula de choque instalada. En efecto, la presión de anular aumentó considerablemente llegando a valores por encima de los 300 psia, afectando el comportamiento de la presión en cabeza la cual empezó a caer drásticamente desde 225 psia hasta llegar a aproximadamente 115 psia, indicando un bloqueo de la bomba. Luego de abrir la válvula de choque y permitir que la presión de anular disminuyera hasta un valor de aproximadamente 120 psia se observó una recuperación en el valor de presión de tubería, que alcanzó un valor de 210 psia.

Por otra parte, se observa que la presión de tubería oscila entre 190 y 210 psia durante el periodo del 29 y 31 de mayo, lo cual indica que el fluido no contaba con suficiente presión para entrar al múltiple de producción que opera a una presión de 220 psia. Por lo tanto, se procede a variar la presión de anular para encontrar el valor en el cual la presión de tubería se estabiliza alcanzando un valor superior a los 220 psi (Figura 10). 


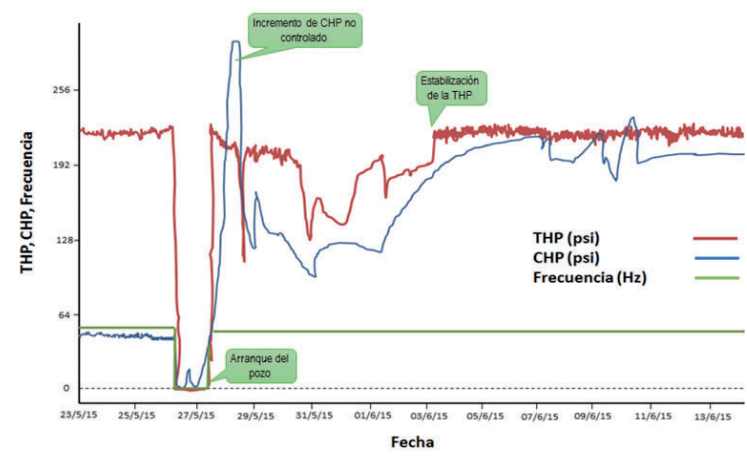

Figura 9. Comportamiento de la THP y CHP del pozo CIRA-M después de implementar el procedimiento propuesto

Se identificó que después que la presión de anular alcanzara un valor de 180 psia y sin modificar la frecuencia de la bomba electro-sumergible, la presión en la tubería aumentó hasta 225 psia, estabilizándose en este valor con oscilaciones muy pequeñas. Es decir, que solo modificando la presión de anular, la presión del fluido en cabeza aumentó, lo cual apoya la hipótesis de que es necesario mantener una presión en fondo determinada para evitar la liberación excesiva de gas y de esta manera permitir que el aceite se desplace sin restricciones desde la formación hacia la cara del pozo. En consecuencia, se estableció que el rango óptimo de operación de la presión de anular para el pozo CIRA-M se encuentra entre 175 y 210 psia.

Otra variable que es importante mencionar y que mejoró notablemente fue la sumergencia efectiva del pozo, lo que implica un aumento en la presión de fondo fluyente. De igual forma, el porcentaje de columna liquida en el espacio anular también se incrementó lo cual evidencia que efectivamente hay una menor liberación de gas. (Tabla 1).

Tabla 1. Sumergencia efectiva y porcentaje de columna liquida antes de implementar el procedimiento.

\begin{tabular}{|cccc|}
\hline Fecha & FLAP (ft) & \% C. liquida & CHP (psia) \\
\hline $20 / 11 / 2014$ & 3 & 24 & 73,5 \\
\hline $25 / 01 / 2015$ & 0 & 25 & 64,1 \\
\hline $03 / 02 / 2015$ & 0 & 100 & 153,9 \\
\hline $13 / 02 / 2015$ & 0 & 90 & 151,9 \\
\hline $17 / 02 / 2015$ & 79 & 100 & 150,7 \\
\hline $21 / 03 / 2015$ & 178 & 28 & 92 \\
\hline $24 / 04 / 2015$ & 194 & 27 & 51,2 \\
\hline $15 / 05 / 2015$ & 221 & 28 & 47,5 \\
\hline $21 / 05 / 2015$ & 220 & 29 & 45,2 \\
\hline $22 / 05 / 2015$ & 185 & 28 & 47,6 \\
\hline $26 / 05 / 2015$ & 383 & 67 & 48,1 \\
\hline
\end{tabular}

Posteriormente, se puede evidenciar detalladamente la mejoría en el comportamiento de la sumergencia efectiva promedio, donde luego de la implementación del procedimiento en el pozo CIRA-M alcanzó un valor de 724 pies, presentando un aumento significativo de 550 pies con respecto a la sumergencia promedio registrada en el periodo abarcado entre los meses de noviembre hasta mayo. (Tabla 2).

Tabla 2. Sumergencia efectiva y porcentaje de columna liquida luego de implementar el procedimiento.

\begin{tabular}{|cccc|}
\hline Fecha & FLAP $(\mathbf{f t})$ & \% C. liquida & CHP (psia) \\
\hline $01 / 06 / 2015$ & 868 & 83 & 127 \\
\hline $02 / 06 / 2015$ & 786 & 84 & 167 \\
\hline $03 / 06 / 2015$ & 692 & 82 & 193 \\
\hline $04 / 06 / 2015$ & 701 & 84 & 205 \\
\hline $05 / 06 / 2015$ & 708 & 84 & 216 \\
\hline $06 / 06 / 2015$ & 671 & 81 & 221 \\
\hline $07 / 06 / 2015$ & 742 & 85 & 194 \\
\hline $08 / 06 / 2015$ & 628 & 78 & 207 \\
\hline $09 / 06 / 2015$ & 720 & 83 & 190 \\
\hline $11 / 06 / 2015$ & 686 & 81 & 202 \\
\hline $13 / 06 / 2015$ & 660 & 77 & 203 \\
\hline $15 / 06 / 2015$ & 726 & 84 & 196 \\
\hline $17 / 06 / 2015$ & 683 & 77 & 191 \\
\hline $19 / 06 / 2015$ & 716 & 82 & 200 \\
\hline $21 / 06 / 2015$ & 680 & 79 & 202 \\
\hline $26 / 06 / 2015$ & 921 & 98 & 188 \\
\hline
\end{tabular}

Como resultado se obtuvo que la producción promedio del pozo CIRA-M fue de 150 barriles por día con un corte de agua del $2 \%$, es decir, se registró un incremento de 50 barriles por día con respecto a la última medición desarrollada antes de aplicar el procedimiento.

\section{VALIDACIÓN DE RESULTADOS POR MEDIO DEL SOFTWARE PIPESIM}

Con el fin de corroborar los datos obtenidos en campo se realizaron simulaciones utilizando los softwares AWP2000 y PIPESIM para de determinar el rango óptimo de la presión de anular en el pozo CIRA-M.

\subsection{DETERMINACIÓN DE LA PRESIÓN DE FONDO}

Mediante el software AWP2000, se calcularon las condiciones de fondo de pozo y entrada a la bomba, a partir de la información recolectada por la empresa operadora con el objetivo de desarrollar un modelo 
de simulación representativo del pozo CIRA-M, los datos de entrada provienen de reportes de producción, registros Sonolog, datos PVT del fluido y del estado mecánico del pozo.

\subsubsection{AJUSTE Y VALIDACIÓN DEL MODELO}

Con el fin de obtener valores confiables en la simulación, se realizó una comparación con los valores obtenidos en los reportes para los meses de Febrero, Marzo, Abril y Mayo, obteniendo el siguiente comportamiento: (Figura 11).

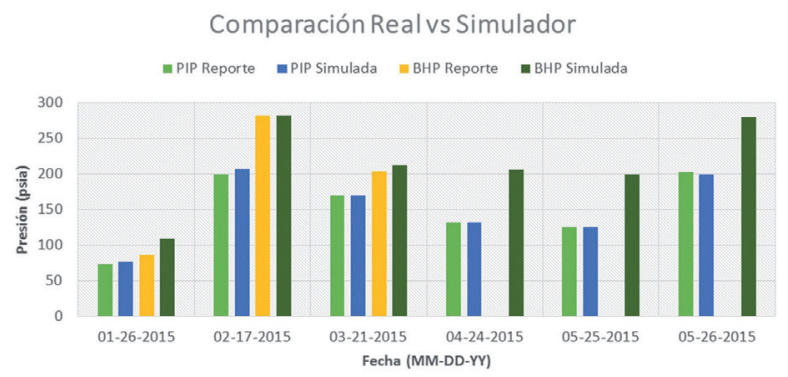

Figura 10. Comparación de BHP y PIP calculada vs simulada

Con base en esto, se puede evidenciar que los parámetros calculados se ajustan al comportamiento real del pozo e independientemente de no contar con la información referente a la presión de fondo fluyendo (BHP) para fechas posteriores al mes de abril debido al dañó del sensor de fondo, se puede utilizar la herramienta software para calcularla y de ese modo obtener un valor de entrada para la segunda etapa de simulación.

\subsubsection{CÁLCULO DE LAS PRESIONES DE FONDO}

Después de corroborar y ajustar el modelo, se llevó a cabo el cálculo de la presión de fondo fluyendo y presión de entrada a la bomba para todos los días del mes de junio, para esto se utilizaron los datos obtenidos de registros acústicos luego de implementar el procedimiento en el pozo (Anexo 1). Se pudo observar que después de realizada la operación de llenado del espacio anular, se estabilizó la presión de anular (CHP) mejorando el balance de presiones a través del sistema de producción y por ende la productividad del pozo.

Después de analizar cada una de las variables de producción, se encontró que para los días entre el 8 de junio y el 21 de junio, el pozo presentaba un comportamiento estable. Por medio de esto, se puede asumir un comportamiento promedio del pozo durante todo el mes. (Tabla 3).

Tabla 3. Condiciones promedio del pozo durante el mes de junio

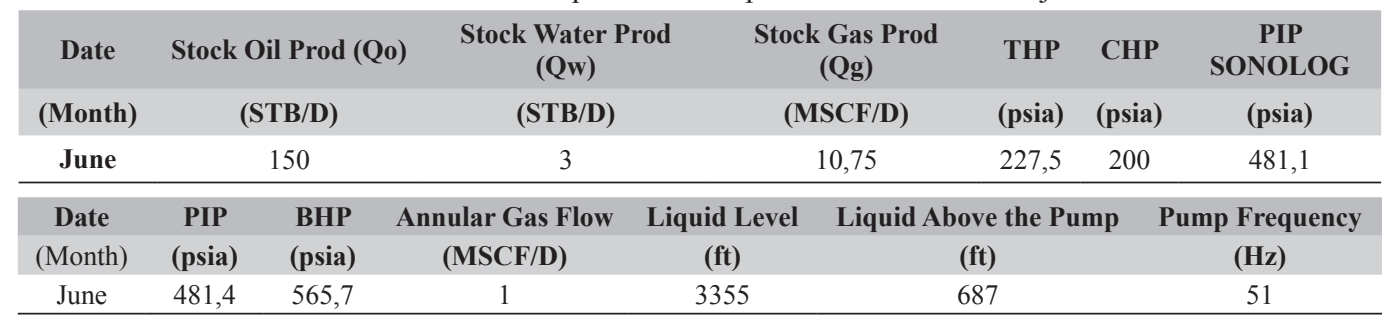

\subsubsection{SENSIBILIZACIÓN DE LA PRESIÓN DE CASING}

Teniendo las condiciones estabilizados del pozo CIRA-M durante el mes de junio, se procede a sensibilizar la presión de anular (CHP), con el fin de estudiar el impacto sobre las condiciones de fondo, siendo esto un parámetro determinante al momento de desarrollar el análisis nodal en el sistema de producción.

Una de las consideraciones durante la sensibilización, es de asumir un comportamiento constate del nivel de líquido sobre la bomba en el espacio anular, ya que este es una variable de entrada al software y no se puede analizar su variación con respecto a cambios en superficie. (Anexo 2).

\subsection{DETERMINACIÓN DE LAS PRESIONES DE ANULAR Y CABEZA}

Una de las condiciones más importante que debemos tener en cuenta a la hora de seleccionar el rango de presión de anular óptima (CHP), es la presión de cabeza de pozo (THP). Para determinarla, fue necesario realizar un modelo de pozo en el software PIPESIM y posteriormente generar el análisis nodal. La información necesaria para llevar a cabo este modelo, fue la misma utilizada en el software AWP 2000. 
Después de ingresar toda la información al software PIPESIM, se debe generar una sensibilización del caudal de líquido producido con el fin de encontrar las condiciones de fondo y cabeza que representen el comportamiento del pozo. (Figura 12).

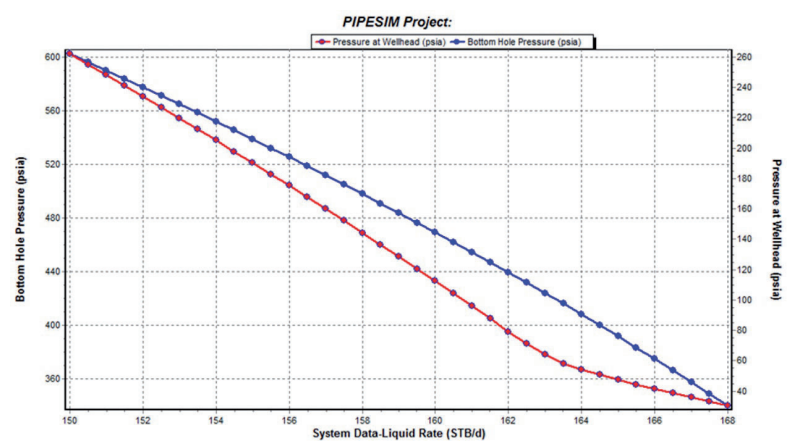

Figura 11. Determinación de la THP y BHP

Finalmente, teniendo la información del comportamiento del pozo, se desarrolla el análisis nodal a través del sistema de producción. Esto con el fin de evaluar los caudales producidos a diferentes presiones de anular y su efecto sobre las presiones de cabeza, entrada a la bomba y fondo de pozo (Figura 13).

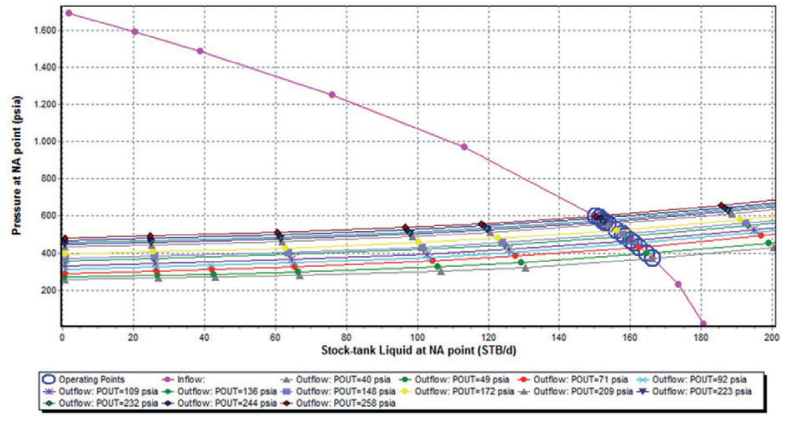

Figura 12. Análisis nodal con sensibilización de la presión de anular

Finalmente, se presentan los datos obtenidos para las diferentes variables de producción luego de variar el valor de presión de anular (CHP) entre 100 y 230 psia, tomando como base el comportamiento mostrado durante el mes de junio, luego de que se implementara el procedimiento en el pozo. (Tabla 4).

Tabla 4. Condiciones de óptimas de operación del pozo CIRA-M

\begin{tabular}{|c|c|c|c|c|c|}
\hline $\begin{array}{c}\text { Casing Head } \\
\text { Pressure (CHP) }\end{array}$ & $\begin{array}{c}\text { Tubing Head } \\
\text { Pressure (THP) }\end{array}$ & $\begin{array}{l}\text { Pump Intake } \\
\text { Pressure } \\
\text { (PIP) }\end{array}$ & $\begin{array}{c}\text { Bottom } \\
\text { Hole Pressure } \\
\text { (BHP) }\end{array}$ & $\begin{array}{l}\text { Stock-Tank } \\
\text { Oil Prod } \\
\text { (Qo) }\end{array}$ & $\begin{array}{c}\text { Stock-tank } \\
\text { Gas Prod } \\
\text { (Qg) }\end{array}$ \\
\hline (psia) & (psia) & (psia) & (psia) & (STB/D) & (MSCF/D) \\
\hline 120 & 92 & 373 & 450 & 158,0 & 12,6 \\
\hline 130 & 109 & 387 & 466 & 157,0 & 12,6 \\
\hline 140 & 136 & 408 & 490 & 155,4 & 12,4 \\
\hline 150 & 148 & 420 & 501 & 154,6 & 12,4 \\
\hline 170 & 172 & 441 & 523 & 153,1 & 12,2 \\
\hline 190 & 209 & 471 & 556 & 150,7 & 12,1 \\
\hline 200 & 223 & 483 & 568 & 149,7 & 12,0 \\
\hline 210 & 232 & 492 & 576 & 149,1 & 11,9 \\
\hline 220 & 244 & 503 & 586 & 148,3 & 11,9 \\
\hline 230 & 258 & 514 & 598 & 147,3 & 11,8 \\
\hline
\end{tabular}

Para determinar el rango óptimo de operación es importante tener en cuenta como se mencionaba anteriormente que el múltiple de producción al que llega el fluido del pozo tiene una presión de entrada de 220 psia y por ende la presión en cabeza es la variable que permite establecer cuál es el rango de presión de anular que permite la entrada de fluido al múltiple. Por lo tanto, el rango óptimo de presión de anular se encuentra entre 200 y 220 psia, ya que permite que la presión en cabeza alcance valores entre 223 y 244 psia, suficiente para facilitar la entrada de fluidos al múltiple de producción.
Los resultados obtenidos en ambos procedimientos (práctico y teórico) muestran que efectivamente controlando la presión de anular se puede evitar el bloqueo por gas y que además existe un rango óptimo de esta presión en el cual la presión en cabeza del pozo alcanza el valor requerido para que el fluido pueda ingresar al múltiple de producción. (Anexo 3). En el procedimiento practico se obtuvo que el rango de presión de anular varía entre 175 y 210 psi mientas que a través de simulación estática se obtuvo que el rango se encuentra entre 200 y 220 psi. Como se puede observar son valores muy cercanos que confirman y validan los resultados obtenidos en el pozo. 


\section{CONCLUSIONES}

La metodología desarrollada permitió identificar que el bloqueo por gas en la cara de la formación es un problema que se presenta actualmente en el campo La Cira-Infantas y del cual no se tenía previo conocimiento.

La falta de supervisión y ajuste de las válvulas de superficie sumados al afán de aumentar la producción de los pozos sin tener en cuenta las características del fluido y los parámetros óptimos de operación podrían llevar a pérdidas de producción importantes.

El ajuste de la presión de anular demostró ser un método eficaz para controlar la presión de fondo y de esta manera evitar que se genere bloqueo por gas en la cara de la formación, además de aumentar la producción del pozo.

La creación de un modelo ajustado de pozo mediante simulación estática, es un método efectivo para recrear los fenómenos que ocurren diariamente en el pozo, brindando datos que pueden ser utilizados para tomar decisiones y establecer criterios operaciones que puedan evitar pérdidas de producción ocasionadas por la alta liberación de gas.

\section{REFERENCIAS}

1. TORRES, D.E; SHARMA, M.M; POPE, G.A; AHMADI, M; MCCULLEY, C.A; LINNEMEYER, H.C; GILANI, S.F. A novel chemical treatment to enhance well productivity in volatile oil reservoirs. SPE 138124. Alberta, Canadá. 2010.

2. TORRES L.D. Enhancing the productivity of volatile oil reservoirs using fluorinated chemical treatments. Trabajo de Grado. Universidad de Texas. Austin, Estados Unidos. 2011.

3. SANNI, M. \& GRINGARTEN, A.C., Well Test Analysis in Volatile Oil Reservoirs. SPE 116239. Denver, Estados Unidos. 2008.

4. HANSSEN, J.E. \& HAUGUM, P., Gas blockage by nonaqueous foams. SPE 21002. Anaheim, Estados Unidos. 1991.

Recepción: 17 de junio de 2016

Aceptación: 31 de octubre de 2016 